\title{
Deficiency of Linolenic Acid in Lefad7 Mutant Tomato Changes the Volatile Profile and Sensory Perception of Disrupted Leaf and Fruit Tissue
}

\author{
Mauricio A. Cañoles ${ }^{1}$ and Randolph M. Beaudry ${ }^{2}$ \\ Department of Horticulture, Michigan State University, East Lansing, MI 48824 \\ Chuanyou $\mathrm{Li}^{3}$ and Gregg Howe ${ }^{4}$ \\ Department of Energy-Plant Research Laboratory, Michigan State University, East Lansing, MI 48824
}

\begin{abstract}
ADDITIONAL INDEX WORDS. fatty acid, linolenic, desaturase, aroma, quality, Lycopersicon esculentum
Abstract. Six-carbon aldehydes and alcohols formed by tomato (Lycopersicon esculentum Mill.) leaf and fruit tissue following disruption are believed to be derived from the degradation of lipids and free fatty acids. Collectively, these C-6 volatiles comprise some of the most important aroma impact compounds. If fatty acids are the primary source of tomato volatiles, then an alteration in the fatty acid composition such as that caused by a mutation in the chloroplastic omega-3-fatty acid desaturase ( $\omega$-3 FAD), referred to as LeFAD7, found in the mutant line of 'Castlemart' termed Lefad7, would be reflected in the volatile profile of disrupted leaf and fruit tissue. Leaves and fruit of the Lefad7 mutant had $\approx 10 \%$ to $15 \%$ of the linolenic acid (18:3) levels and about 1.5- to 3-fold higher linoleic acid (18:2) levels found in the parent line. Production of unsaturated C-6 aldehydes $Z$-3-hexenal, $Z$-3-hexenol, and $E$-2-hexenal and the alcohol Z-3-hexenol derived from 18:3 was markedly reduced in disrupted leaf and fruit tissue of the Lefad7 mutant line. Conversely, the production of the saturated C-6 aldehyde hexanal and its alcohol, hexanol, were markedly higher in the mutant line. The shift in the volatile profile brought about by the loss of chloroplastic FAD activity in the Lefad7 line was detected by sensory panels at high significance levels $(P<0.0005)$ and detrimentally affected fruit sensory quality. The ratios and amounts of $\mathrm{C}-6$ saturated and unsaturated aldehydes and alcohols produced by tomato were dependent on substrate levels, suggesting that practices that alter the content of linoleic and linolenic acids or change their ratios can influence tomato flavor.
\end{abstract}

Perception of aroma is a very complex process involving both detection and cognitive integration (Baldwin et al., 2000). Given that the aroma of most consumables is a function of many volatiles, assessing the influence of a single volatile is a challenge, and developing strategies to improve aroma is, perhaps, even more problematic.

Of the more than 400 volatiles reported for tomato fruit (Frenkel and Jen, 1989; Grierson and Kader, 1986; Tandon et al., 2000), only 30 are present at a concentration higher than $1 \mathrm{~nL} \cdot \mathrm{L}^{-1}$. Of those, only 16 have a positive log odor unit, which is defined as the logarithm of ratio of the concentration of an aroma volatile to its odor threshold (Buttery, 1993). A log odor unit of 0 indicates that the odorant is present at its threshold level; log odor units of 1 and -1 would indicate the odorant is at 10 times and 1/10th the threshold level, respectively. Reducing the production of those volatiles with positive log odor units, increasing the production of those volatiles with negative log odor units, or changing the ratios of impact compounds have the potential to alter sensory perception of tomato leaves and/or fruit.

Lipids and fatty acids (FA) are thought to act as precursors to the aldehydes $Z$-3-hexenal, hexanal, and E-2-hexenal, the alcohols 1-hexanol and Z-3-hexenol, and the ketone 1-penten-3-one

Received for publication 9 Nov. 2004. Accepted for publication 21 Sept. 2005. We thank Gustavo Bonaventure and John Ohlrogge for fatty acid analysis. Research was supported by MIDEPLAN, Gobierno de Chile, and the Michigan Agricultural Experiment Station. Use of trade names does not imply endorsement of the products named or criticism of similar ones not named.

1 PhD Student.

${ }^{2}$ Professor; to whom reprint requests should be addressed; e-mail address: beaudry@msu.edu

${ }^{3}$ Postdoctoral Research Associate.

${ }^{4}$ Associate Professor.
(Baldwin et al., 2000), which provide some of the most important flavor notes to tomato (Table 1). Our current understanding is that the formation of these compounds involves the action of a sequence of enzymes [lipase, lipoxygenase (LOX), hydroperoxide lyase (HPL), isomerase, and alcohol dehydrogenase (ADH)] on glycerolipids containing the fatty acids linoleic acid (18:2) and linolenic acid (18:3) (Bate et al., 1998; Galliard and Matthew, 1977; Riley et al., 1996). FA composition of tomato is variable

Table 1. Odor descriptors, fatty acid precursor, and log odor units for C-6 and C-5 volatiles from lipid oxidation in tomato fruit.

\begin{tabular}{lllc}
\hline $\begin{array}{l}\text { Aroma } \\
\text { compound }\end{array}$ & \multicolumn{1}{c}{$\begin{array}{c}\text { Odor } \\
\text { descriptor }\end{array}$} & $\begin{array}{l}\text { Fatty acid } \\
\text { precursor }\end{array}$ & $\begin{array}{c}\text { Log odor } \\
\text { units }\end{array}$ \\
\hline Z-3-Hexenal & Tomato, citrus & $\begin{array}{l}\text { Linolenic acid } \\
(18: 3)\end{array}$ & 3.7 \\
Hexanal & Stale, grassy, green & $\begin{array}{l}\text { Linoleic acid } \\
(18: 2)\end{array}$ & 2.8 \\
E-2-Hexenal & Stale, green, vine & $\begin{array}{l}\text { Linolenic acid } \\
(18: 3)\end{array}$ & 1.2 \\
1-Hexanol & Glue, oil & $\begin{array}{l}\text { Linoleic acid } \\
(18: 2)\end{array}$ & -1.9 \\
Z-3-Hexenol & Green, celery & $\begin{array}{l}\text { Linolenic acid } \\
(18: 3)\end{array}$ & 0.3 \\
1-Penten-3-one & Fresh, sweet & $\begin{array}{l}\text { Linolenic acid } \\
(18: 3)\end{array}$ & 2.7 \\
2-Pentenal & Stale, oil & $\begin{array}{l}\text { Linolenic acid } \\
(18: 3)\end{array}$ & -1.0 \\
1-Penten-3-ol & Pungent, green, fruity & $\begin{array}{l}\text { Linolenic acid } \\
(18: 3)\end{array}$ & -0.6 \\
& & r. &
\end{tabular}

zTandon et al., 2000

yButtery, 1993. 
and dependent on the cultivated variety, but in general the most abundant fatty acids are 18:3 and 18:2, which account for $60 \%$ to $70 \%$ of the total FA content (Gray et al., 1999; Li et al., 2003; Wang et al., 1996).

Substrate feeding experiments using protein extracts or homogenates from fruit demonstrated that the addition of 18:2 enhances the production of hexanal and 1-hexanol, and addition of 18:3 enhances Z-3-hexenal, $E$-2-hexenal, and Z-3-hexenol formation (Boukobza et al., 2001; Galliard and Matthew, 1977; Riley and Thompson, 1998). Somewhat paradoxically, no strong relationship was found between LOX, HPL, and ADH activities and the amount of volatiles produced (Yilmaz et al., 2001), suggesting that improving volatile formation is a complex goal that may not be achievable by modifying a single step in the LOX pathway.

Gray et al. (1999) measured the amounts of saturated and unsaturated C-6 aldehydes produced by tomato cultivars that differ in amount of 18:2 and 18:3. They found a significantly higher hexanal to hexenal ratio for tomato fruit in which the ratio of 18:2 to $18: 3$ was higher. Wang et al. (1996) found similar results using a transgenic approach to alter LOX substrate levels by constitutive expression of a yeast delta- 9 desaturase gene. The amounts of $16: 1,18: 1$, and 18:2 in the fruit were significantly increased as was the total amount of FA. The amount of 18:3 was slightly reduced relative to the wild type. The volatiles hexanal and 1-hexanol, presumed to be derived from 18:1 and 18:2, were enhanced $267 \%$ and $407 \%$, respectively, relative to untransformed tomato. However, three of four C-6 volatiles presumed to be derived from 18:3 also increased to a similar extent in the transformed plant material, as did several non-lipid-derived volatiles, thus raising doubts as to the interpretation of the data. A clearer picture of the relationship between endogenous fatty acid content and fruit and leaf volatiles may be derived using tomato lines possessing more dramatic shifts in fatty acid content.

One such tomato line is the fatty acid mutant identified by Howe and Ryan (1999), based on its impaired ability to respond to wounding. The mutant, spr 2 (suppressed in 35S::prosystemin-mediated responses), was subsequently identified as being deficient in jasmonic acid (JA) biosynthesis (Li et al., 2002) due to considerably lower levels of 18:3 than wild type (Li et al., 2003). It has been recently demonstrated that the Spr 2 gene of tomato encodes a chloroplastic $\omega$-3 FAdesaturase (Li et al., 2003), now called LeFAD7. The mutation of the $\omega-3$ FAD was shown to reduce the linolenic acid content in leaves, but did not affect root FA content. Fruit FA content was not reported.

We hypothesized that an alteration in the fatty acid composition caused by the spr 2 mutation (renamed Lefad7) would be reflected in the volatile profile of disrupted leaf and fruit tissue. The current work analyzes the production of C- 6 volatiles by tomato leaves and fruit at different developmental stages by two lines differing in fatty acid composition, and how this change in the volatile profile is perceived by a non-trained sensory panel.

\section{Materials and Methods}

Plant material. 'Castlemart' tomato and the mutant of this cultivar, Lefad7, obtained by ethyl methanesulfonate mutagenesis (EMS) (Howe and Ryan, 1999), were grown in a greenhouse under a constant temperature of $20^{\circ} \mathrm{C}$. Young (50\% expanded) and mature (fully expanded) leaves and immature green and mature red fruit were sampled for FA and volatile analysis.

FATTY ACID Composition. Total lipids were extracted from $400 \mathrm{mg}$ of leaf and $500 \mathrm{mg}$ of fruit tissue with two 6-mL aliquots of hexane and once with $4 \mathrm{~mL}$ of 2:7 (v:v) isopropanol:hexane. Esterification/transesterification of FA was performed with methanolic: $\mathrm{H}_{2} \mathrm{SO}_{4}(2.5 \%)$ to generate fatty acid methyl esters (FAMES) (Conconi et al., 1996). FAMES were analyzed by gas chromatography (GC) with a DB-23 capillary column (J\&W Scientific, Folsom, Calif.) and flame ionization detector (FID) as described by Bonaventure et al. (2003).

Evaluation of AROMA compounds. For leaf volatile analysis, one leaf disk (35 mg) from each experimental unit was crushed in a $20-\mathrm{mL}$ amber glass vial and sealed. After $3 \mathrm{~min}$, when volatile production is essentially complete (Boukobza et al., 2001), 1 $\mathrm{mL}$ of $50 \% \mathrm{CaCl}_{2}$ solution was added to stop further enzymatic reactions. The vial was immediately closed with a Mininert valve (Supelco, Bellefonte, Pa.), and stored at $-20{ }^{\circ} \mathrm{C}$ until volatile analysis. For volatile analysis of the homogenized leaf tissue, the homogenate was thawed and the vial heated and sampled as described below. For fruit volatile analysis, $10 \mathrm{~g}$ of pericarp tissue were homogenized in $5 \mathrm{~mL}$ of distilled water using a tissue homogenizer (PT 10/35; Brinkmann Instrument Co., Westbury, N.Y.). After $3 \mathrm{~min}$ in a closed $50-\mathrm{mL}$ tube, $6 \mathrm{~mL}$ of $50 \% \mathrm{CaCl}_{2}$ $(\mathrm{w} / \mathrm{v})$ solution was added and cooled immediately in a $-80{ }^{\circ} \mathrm{C}$ freezer and held until analysis. Before volatile analysis, the homogenate was thawed and as soon as ice was no longer present, $5 \mathrm{~mL}$ of the homogenate were placed in a 20-mL amber glass vial and closed with a valved septum (Mininert valve; Supelco).

Vials containing homogenate were placed in a water bath held at $37{ }^{\circ} \mathrm{C}$ for $30 \mathrm{~min}$ before analysis. Volatile compounds were extracted from the headspace using a solid-phase microextraction (SPME) fiber (65 $\mu \mathrm{m}$ PDMS-DVB; Supelco) similar to the method of Song et al. (1998). The fiber was held in the vial for 3 min to allow absorption of volatile compounds. A gas chromatograph (HP 6890 Series GC; Hewlett-Packard Co., Wilmington, Del.) was used for analyte separation, and a time-of-flight mass spectrometer (Pegasus II; Leco, St. Joseph, Mich.) was used for analyte detection, identification, and quantification. The GC column (SupelcoWax-10; Supelco) was $30 \mathrm{~m}$ long $\times 0.2 \mathrm{~mm}$ i.d. with a $0.2 \mu$ m-thick coating. The GC oven was heated from 40 to 200 ${ }^{\circ} \mathrm{C}$ at a rate of $50{ }^{\circ} \mathrm{C} / \mathrm{min}$ and held at $200{ }^{\circ} \mathrm{C}$ for $3 \mathrm{~min}$. In order to quantify the headspace concentration of hexanal, Z-3-hexenal, E-2-hexenal, 1-hexanol, and Z-3-hexenol, gas standards were created using authenticated compounds according to the method of Song et al. (1998). For leaf analysis, $0.25 \mu \mathrm{g}$ of 2-octanone (10 $\mu \mathrm{L}$ of a $25 \mathrm{ng} \cdot \mu \mathrm{L}^{-1}$ solution in water) was added in the vial as internal standard. For fruit samples, $0.4 \mu \mathrm{g}$ of 2-octanone was added to the vial.

Sensory evaluation. A sensory test was performed to determine if the aroma of the crushed tissues for mutant line differed from the wild type line. For leaves, five leaf disks ( $\approx 170 \mathrm{mg})$ were crushed in a $20-\mathrm{mL}$ vial, closed with Teflon-lined caps, and held at room temperature. One milliliter of a $50 \% \mathrm{CaCl}_{2}$ aqueous solution was added after 3 min to stop enzymatic reactions. Vials were kept at $-20{ }^{\circ} \mathrm{C}$ until the sensory test was performed. For fruit, $30 \mathrm{~g}$ of pericarp were blended and $20 \mathrm{~mL}$ of $50 \% \mathrm{CaCl}_{2}$ solution were added after $3 \mathrm{~min}$. Five milliliters of the homogenate were transferred into $20-\mathrm{mL}$ vials and kept at $-20{ }^{\circ} \mathrm{C}$ until the sensory test.

A triangle test for aroma was performed as indicated by Meilgaard et al. (1999). Panelists were presented with three vials; two vials held the same homogenate and the third held a different homogenate. Panelists were asked to smell each vial and choose which vial scent differed from the other two. The order in which the samples, mutant or wild type, were presented to the panelists 
were arranged so that all six possible combinations were used. There were at least five replications of each sample combination presented to panelists. For leaf analysis, 32 panelists were used; for fruit analysis, there were 36 panelists.

A preference test was performed together with the triangle test for fruit (Meilgaard et al., 1999). The panel was asked if the homogenate they chose as being different from the other two was preferable or not-preferable. The preference test was designed to qualify the sensory differences detected in the triangle test. The hypothesis tested was "more than $50 \%$ of panelists prefer one aroma over the other" (Meilgaard et al., 1999).

Statistical anAlysis. All data for FA content and headspace volatile concentration were expressed as the mean \pm SE. Data were analyzed using one-way analysis of variance by PROC MIXED of SAS (version 8e; SAS Institute, Cary, N.C.). Statistical significance of sensory evaluation data was determined using Table T8 in Meilgaard et al. (1999).

\section{Results}

FAtTy ACID COMPosition. The total FA content of leaves did not differ between wild type and Lefad7 mutant lines, averaging $29.3 \mu \mathrm{mol} \cdot \mathrm{g}^{-1}$ on a fresh weight basis. Of the eight FAs evaluated for mutant and wild type leaves, only dienoic (16:2 and 18:2) and trienoic (16:3 and 18:3) FAs differed (Fig. 1A). Mutant leaf 16:3 content was $1 \%$ of wild type, while the $18: 3$ content was $16 \%$ of wild type. The content of 16:2 and 18:2 in mutant leaves was
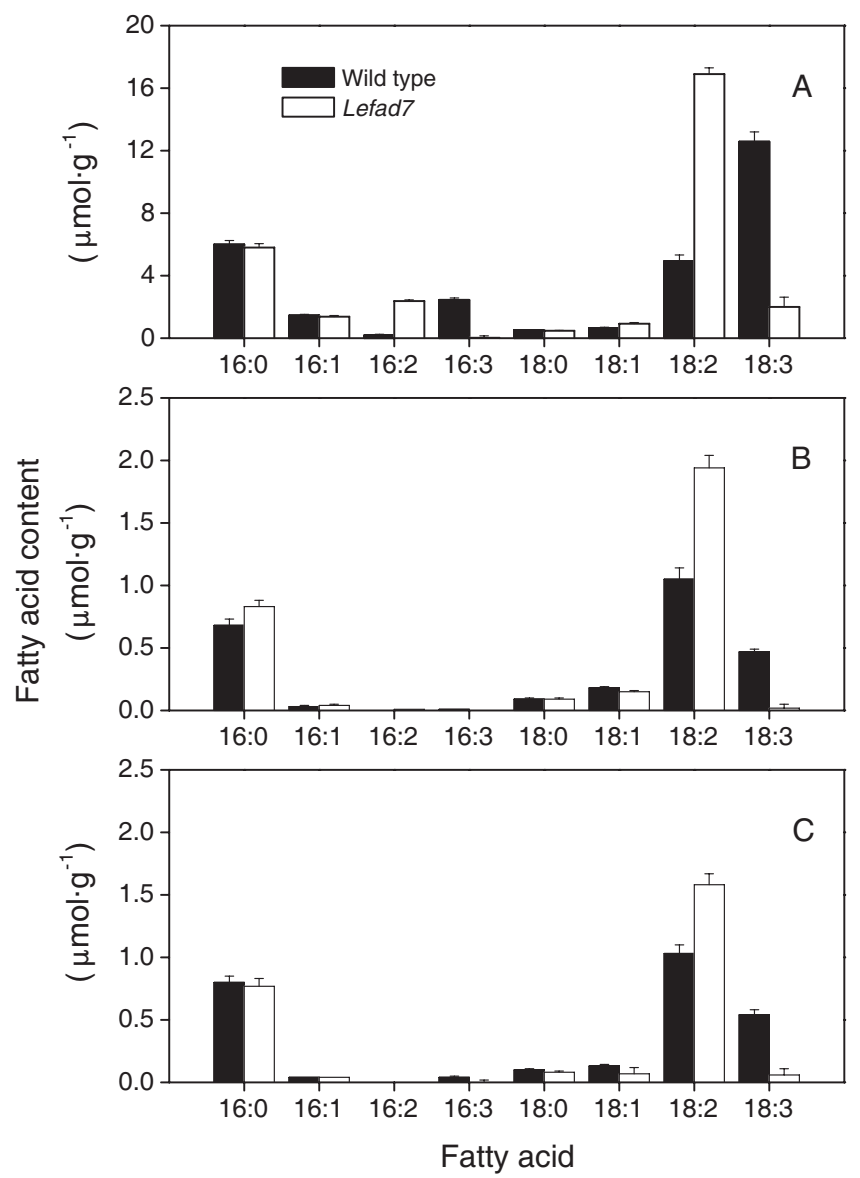

Fig. 1. Content of fatty acids containing 16- and 18-carbons and 0, 1, 2, and 3 unsaturated carbon to carbon bonds in wild type and Lefad7 'Castlemart' tomato tissue on a fresh weight basis for (A) mature leaf, (B) immature green fruit, and (C) mature red fruit. Vertical bars represent SE $(n=4$ to 6$)$.
13- and 3.5-fold higher than in wild type leaves. In the wild type tomato leaf, the most abundant FA was 18:3, constituting about $44 \%$ of total FA while 18:2 constituted 18\%; in Lefad7 leaves, the most abundant was 18:2, comprising about $57 \%$ of the total (data not shown), while 18:3 represented only 7\% of total FA. Octadecanoic acids accounted for $\approx 65 \%$ of total FA. Maturity did not affect total FA content or composition for wild type and mutant leaves (not shown).

As in leaves, the FA composition and total content in fruit was not affected by maturity stage (Fig. 1B-C) for wild type and mutant lines, averaging $2.7 \mu \mathrm{mol} \cdot \mathrm{g}^{-1}$. The content of hexadecanoic acid (16:0) was $\approx 20 \%$ to $30 \%$ of the total FA content and was unaffected by the mutation (data not shown). Tri- and dienoic forms of hexadecanoic acid (16:3 and 16:2) were absent or present in very low amounts in tomato fruit; whereas di- and trienoic forms of octadecanoic acids accounted for more than $60 \%$ of the total FA content (data not shown). The loss of FAD7 activity in the mutant caused the mole fraction of 18:2 to increase from $\approx 40 \%$ to $60 \%$, and caused the mole fraction of 18:3 to decrease from $\approx 20 \%$ to $1 \%$ to $3 \%$ of the total FA content. The impact of Lefad7 mutation on 18:3 content reduction was greater for fruit than for leaves.

Volatile AROMA Compounds. C-6 aldehydes accounted for 99\% of C-6 volatiles derived from the LOX pathway for all the tissues and stages analyzed in wild type and Lefad7 plants (data not shown). The mutation markedly altered the volatile production of the aldehydes hexanal, $Z$-3-hexenal, and $E$-2-hexenal for leaf and fruit tissue (Figs. 2-4).
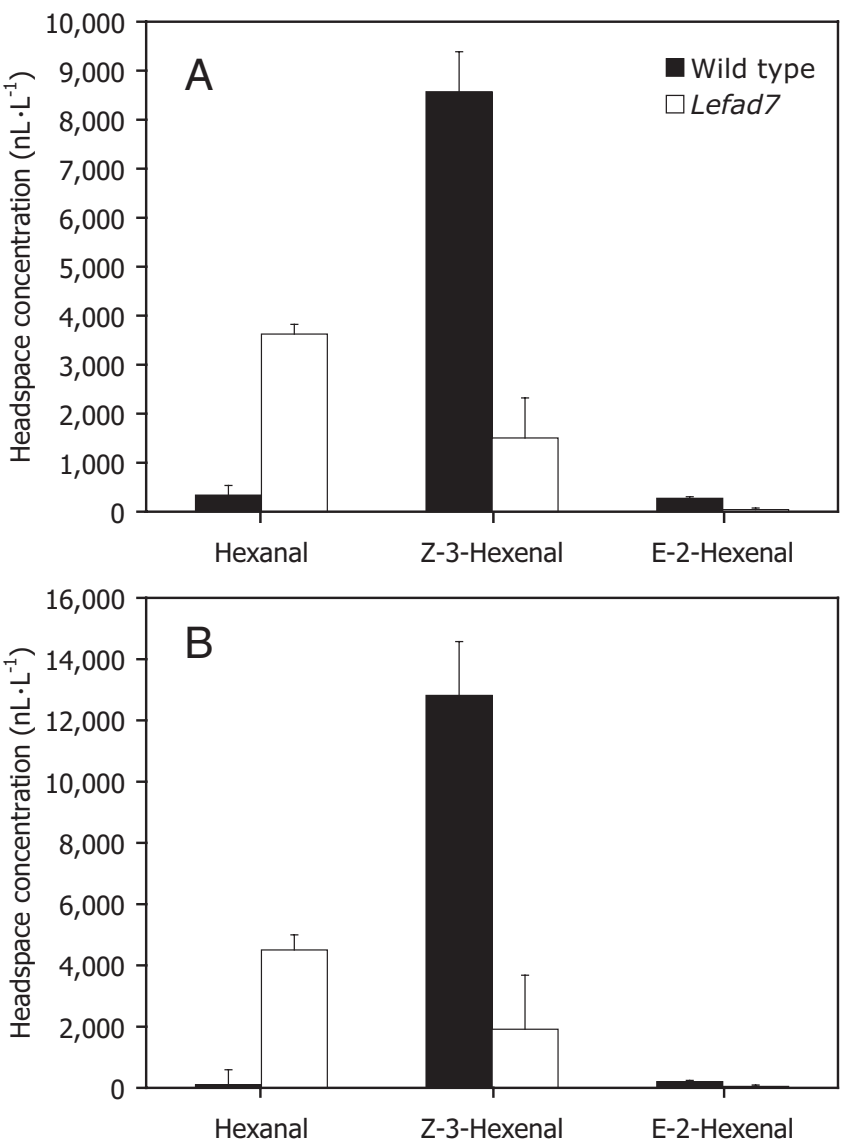

Fig. 2. C-6 aldehyde concentrations in the headspace of vials containing crushed (A) 50\% expanded and (B) fully expanded wild type and Lefad7 'Castlemart' tomato leaves. Vertical bars represent SE $(n=4)$. 

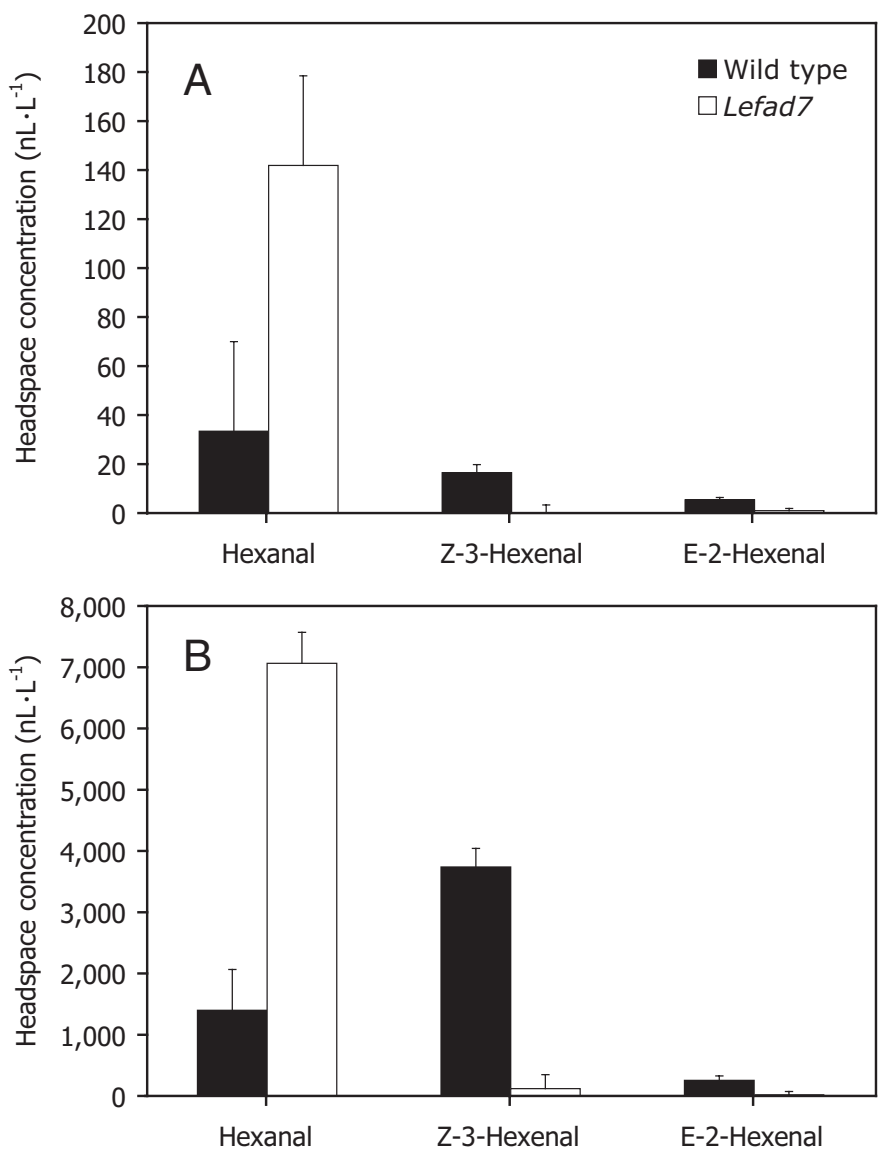

Fig. 3. C-6 aldehyde concentrations in the headspace of vials containing homogenized (A) immature green and (B) mature red wild type and Lefad7 'Castlemart' tomato fruit. Vertical bars represent SE $(n=4$ to 6$)$.

In wild type leaves, the most abundant C-6 aldehyde was Z-3-hexenal (93\%); in Lefad7 mutant leaves, the most abundant C-6 aldehyde was hexanal (70\%) (Fig. 2A-B). In mutant leaves, Z-3-hexenal accounted for only $29 \%$ of the total C-6 aldehydes. For wild type and mutant leaves, the concentration of $E$-2-hexenal was low, amounting to $3 \%$ and $1 \%$ of the total, respectively. There was no difference in volatile composition and concentration for young and mature leaves.

In wild type fruit, the most abundant C- 6 aldehyde was hexanal in green fruit and Z-3-hexenal in red fruit (Fig. 3). For Lefad7 fruit, Z-3-hexenal production was suppressed to extremely low levels and hexanal was the predominant C-6 aldehyde for both stages of development (Fig. 3). However, C-6 aldehyde production differed dramatically between developmental stages for wild type and mutant lines. Total C-6 aldehydes for red tomato fruit were 100-fold higher than green fruit for the wild type line and 50-fold higher for the mutant line, with no similar accompanying shift in FA content (Table 2). The greatest relative increase in the aldehydes of wild type fruit occurred for Z-3-hexenal, which increased over 200-fold between immature green and mature red stages. In mutant fruit, this aldehyde was not detected at the green immature stage, but at the red stage it was possible to detect the presence of small amount $\left(120 \mathrm{~nL} \cdot \mathrm{L}^{-1}\right)$.

In terms of relative composition within the C-6 aldehydes, wild type green fruit homogenates produced more hexanal $(60 \%$ of total C-6 aldehydes) than the sum of Z-3- and E-2-hexenals, while red fruit produced a greater proportion $(74 \%)$ of $Z-3-$ and $E$-2-hexenals (Fig. 4). Wild type leaves had a preference for the production of unsaturated aldehydes $(96 \%)$, which more closely resembled the aldehyde profile of mature red fruit. Mutant leaves had a volatile profile that was somewhat depleted of unsaturated C-6 volatiles relative to wild type.

Sensory EValuation. In the triangle test for leaves, 26 out of 32 subjects were able to correctly choose the odd sample between the three vials (Table 3). For fruit, 28 out of 36 subjects were able to differentiate the odd sample from the set of three vials. The results of both triangle tests demonstrated that the difference in volatiles of mutant and wild type leaf and fruit tissues can be readily perceived $(P<0.0005)$.

In the preference test, the tested hypothesis was that more than $50 \%$ of the panel preferred the tomato aroma of wild type fruit. The majority of respondents expressed a preference for the aroma of the wild type tomato fruit homogenate and the hypothesis was accepted $(P<0.0005)$.

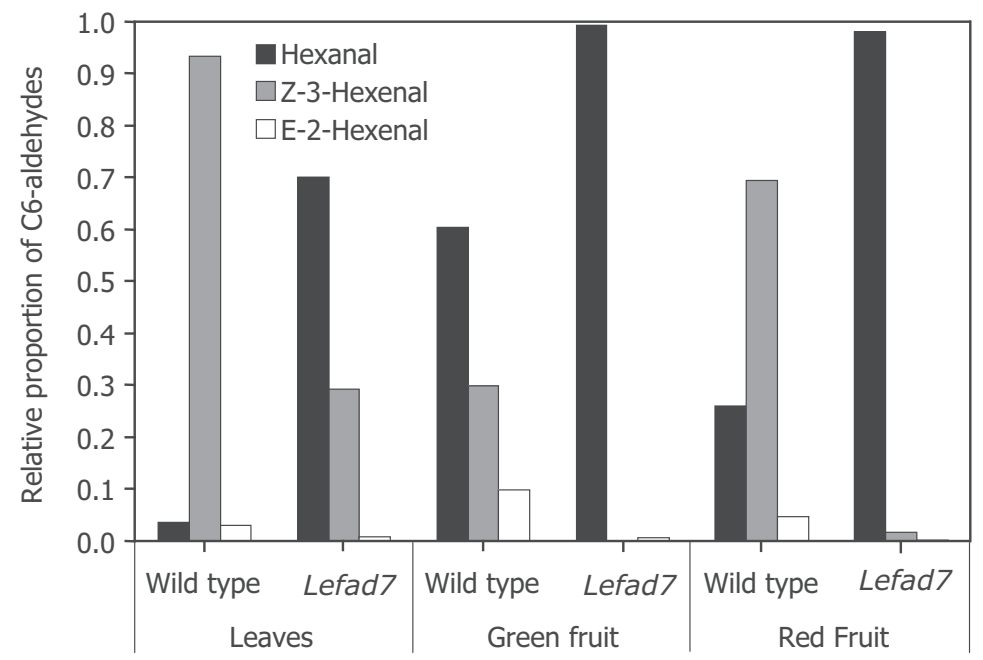

Fig. 4. C-6 aldehyde concentrations in the headspace of vials containing crushed leaf tissue and homogenized immature green and mature red fruit from wild type and Lefad7 'Castlemart' tomato fruit.
Table 2. Ratios of C-6 volatile production and the fatty acid (FA) sources of those volatiles for red mature fruit relative to immature green fruit of wild type and Lefad7 mutant 'Castlemart' tomatoes. Each number represents an average of five determinations; n.d. indicates compound not detected in either red or green fruit.

\begin{tabular}{|c|c|c|c|c|}
\hline \multirow[b]{2}{*}{ Volatile } & \multicolumn{2}{|c|}{ Wild type } & \multicolumn{2}{|c|}{ Lefad7 } \\
\hline & Volatile & $\begin{array}{c}\text { FA } \\
\text { sources }\end{array}$ & Aldehyde & $\begin{array}{c}\text { FA } \\
\text { sources }\end{array}$ \\
\hline Hexanal & 42.3 & 1.03 & 49.7 & 0.83 \\
\hline Z-3-Hexenal & 234 & 1.21 & n.d. & $2.9^{\mathrm{z}}$ \\
\hline$E$-2-Hexenal & 50.8 & 1.21 & 18.0 & $2.9^{z}$ \\
\hline Total C-6 aldehydes & 99.8 & 1.06 & 50.3 & 0.84 \\
\hline 1-Hexanol & n.d. & 1.03 & 3.0 & 0.83 \\
\hline Z-3-Hexenol & 4.0 & 1.21 & n.d. & $2.9^{\mathrm{z}}$ \\
\hline Total C-6 alcohols & 4.0 & 1.06 & 4.0 & 0.84 \\
\hline
\end{tabular}

${ }^{2}$ Low absolute levels of 16:3 and 18:3 reduced the accuracy of this determination. 
Table 3. Correct responses and panel size to determine if non-trained panelists could correctly identify the scent of the odd sample in triangle test comparisons of the aroma of Lefad7 mutant and wild type 'Castlemart' tomatoes. Panelist preference was determined for the 28 panelists correctly identifying the odd sample for fruit.

\begin{tabular}{lccc}
\hline & \multicolumn{2}{c}{ No. of subjects } & \\
\cline { 2 - 3 } & Correct & Total & $P^{\mathrm{z}}$ \\
\hline Triangle test, leaves & 26 & 32 & $<0.0005$ \\
Triangle test, fruit & 28 & 36 & $<0.0005$ \\
& Wild type better & Total & $P$ \\
\cline { 2 - 3 } Preference test, fruit & 23 & 28 & $<0.0005$ \\
\hline
\end{tabular}

${ }^{\mathrm{z}}$ Meilgaard et al., 1999 (Table 8).

\section{Discussion}

The FA composition and content of wild type and mutant leaves in this study was similar to those previously published (Li et al., 2003). One might have expected that reduced LeFad7 expression would have reduced total FA accumulation. However, Lefad 7 data are consistent with observations for $\omega-3$ FAD mutants of Arabidopsis thaliana (L.) Heynh., specifically, a fad7 mutant (Zhuang et al., 1996) and a fad3-2, fad7-2, fad8 triple mutant (Routaboul et al., 2000), for which the total FA content is not affected by the lack of $\omega$-3 FAD activity. The lack of an impact on total FA content by a loss of $\omega-3$ FAD activity contrasts with results of overexpression of a yeast-derived delta- 9 desaturase in tomato, in which fruit FA content was increased $\approx 100 \%$ in fruit (Wang et al.,1996) and 25\% in leaves (Wang et al., 2001). However, the delta-9 desaturase forms dienoic fatty acids, which act as substrates for $\omega-3$ FAD. It is possible that FA content is controlled at a point in the pathway prior to $\omega-3$ FAD.

The reduction of 18:3 in tomato vegetative and reproductive tissue and the accompanying dramatic decrease in the concentration of the aldehyde and alcohol volatiles derived from its peroxidation confirm that $18: 3$ is the primary precursor to $Z$-3-hexenal, $E$-2hexenal, and $Z$-3-hexenol in tomato, as suggested by Baldwin et al. (2000). In mutant leaves, $Z$-3-hexenal, which accounted for only $29 \%$ of the total C-6 aldehydes, was most likely derived from the residual amounts of $18: 3$ produced by non-chloroplastic FAD (FAD3) as suggested by $\mathrm{Li}$ et al. (2003).

Considering that FA and C-6 volatile composition was not significantly different between young and mature leaves, the data suggest that the regulation of FA oxidation in leaves does not change developmentally as a function of the age of a plant organ. However, in fruit, the 50- to 100-fold increase in C-6 aldehyde production associated with maturation and ripening (Table 2) with no similar accompanying shift in FA content, likely reflects that an increase in LOX pathway activity accompanies fruit ripening, which is consistent with the recent findings of Chen et al. (2004) regarding TomLoxC. Changes in lipase activity could also be affecting the availability of substrate for LOX pathway. However, data for the involvement of lipase are lacking; the only lipase expression analyses during tomato fruit ripening have been done on phospholipase D (PLD) (Jandus et al., 1997; Pinhero et al., 2003; Whitaker et al., 2001). PLD activity increased during ripening process for some cultivars, but decreased or did not change in others.

The ripening-related enhanced LOX pathway activity in fruit is most likely associated with an increase in expression of lipoxygenase genes (Griffiths et al., 1999a). The gene most likely to control fruit LOX activity during ripening is TomLoxC, given that TomLoxD is not expressed in fruit (Heitz et al., 1997), and antisense-mediated silencing of TomLoxA and TomLoxB did not result in a change in volatile composition (Griffiths et al., 1999b). Further, Chen et al. (2004) demonstrated that suppression of TomLoxC, which codes for a chloroplast-targeted lipoxygenase, markedly suppresses the biosynthesis of C-6 aldehydes in tomato homogenates. The impact of TomLoxC on leaf volatiles was not reported.

Another enzyme in the pathway, HPL, is considered to undergo little change in activity during tomato fruit ripening (Riley et al., 1996). However, differences in the relative aldehyde composition (Fig. 4) between the two stages of fruit, indicate a marked increase in the production of 18:3 oxidation products (e.g., Z-3hexenal) without a similar increase in the formation of hexanal from 18:2. This developmental change in substrate preference in the LOX pathway is most likely related to HPL, which has a higher affinity for 13-hydroperoxide of 18:3 (13-HPOT) than 13-hydroperoxide of 18:2 (13-HPOD) (Fauconnier et al., 1997; Howe et al., 2000; Suurmeijer et al., 2000). Our data therefore imply a change in HPL activity in vivo, contrary to the findings of Riley et al. (1996). The large increase in 18:3-derived C-6 volatiles relative to 18:2-derived C-6 volatiles is likely not related to LOX enzymes, which have been shown to have no specificity for 18:2 or 18:3 (Feussner and Wasternack, 2002).

The large increase in C-6 volatile production by ripening wild type tomato fruit relative to immature fruit with no change in FA content or composition suggests that an increase in volatile biosynthesis during ripening is not strictly a function of FA substrate availability. Nevertheless, FA substrate levels did impact the volatile profile since the near elimination of $\omega-3$ FAs as a substrate for the LOX pathway did impact the capacity for synthesis of C-6 volatiles.

The perception of differences in the volatile profile of wild type and Lefad7 leaves and fruit by a sensory panel is the first demonstration that alteration in FA composition under isogenic background can alter the aroma of vegetative or reproductive tissue in tomato. The LeFad7 mutation did not appear to negatively impact LOX pathway performance, thus perceived quality changes are attributable to a single factor, the balance between dienoic and trienoic fatty acids. It is not certain, however, whether panelists were responding to a loss in 18:3-related volatiles or to an increase in 18:2-related volatiles. Likely, a trained panel would be needed to make this determination.

The data for chemical and sensory impacts of the reduction in the activity of LeFAD7 highlight an important feature of manipulating single genes in this and other metabolic pathways. Despite the relative simplicity of the LOX pathway for volatile formation, a single gene change impacts multiple compounds, suggesting that the enhancement of a single desirable compound or class of compounds, may not be achievable. While the minimal unit of change is a single gene, the minimal scope of change is likely to be numerous products of the pathway impacted. Thus, it might be difficult to develop, for instance, tomato lines in which a single volatile compound is enhanced or suppressed.

The current work demonstrated that in vivo reduction of 18:3 content in tomato tissue reduces the biosynthesis of several of the most important volatile compounds in tomato aroma, including the volatile with the highest log-odor units, Z-3-hexenal, but it does not reduce the capacity of C-6 aldehyde and alcohol production by the LOX pathway. The altered concentration of LOX pathway oxidation products, mainly aldehydes, was detected by the olfactory sense of humans, affecting the sensory 
quality of tomato fruit. The current results open the possibility of improvement of tomato fruit sensory quality by changing FA composition. An increase in 18:3 content and/or the rate of oxidation of linolenic acid may result in an increase in the relative concentration of the most important tomato volatile compound, Z-3-hexenal. An increase in 18:3 content may also be attended by increases in non-target compounds such as 1-penten-3-one and other C-5 volatiles from fatty acid degradation, which may also impact fruit quality attributes and may have unintended impacts on physiological processes associated with oxylipins.

\section{Literature Cited}

Baldwin, E.A., J.W. Scott, C.K. Shewmaker, and W. Schuch. 2000. Flavor trivia and tomato aroma: Biochemistry and possible mechanisms for control and important aroma components. HortScience 35(6): 1013-1022.

Bate, N.J., J.C.M. Riley, J.E. Thompson, and S.J. Rothstein. 1998. Quantitative and qualitative differences in C-6 volatile production from the lipoxygenase pathway in an alcohol dehydrogenases mutant of Arabidopsis thaliana. Physiol. Plant. 104:97-104.

Bonaventure, G., J.J. Salas, M.R. Pollard, and J.B. Ohlrogge. 2003. Disruption of the FATB gene in Arabidopsis demonstrates an essential role of saturated fatty acids in plant growth. Plant Cell 15(4):1020-1033.

Boukobza, F., P.J. Dunphy, and A.J. Taylor. 2001. Measurement of lipid oxidation-derived volatiles in fresh tomatoes. Postharvest Biol. Technol. 23:117-131.

Buttery, R.G. 1993. Quantitative and sensory aspects of flavor of tomato and other vegetables and fruits, p. 259-286. In: T.E. Acree and R. Teranishi (eds.). Flavor science: Sensible principles and techniques, Chapter 8. Amer. Chem. Soc., Washington, D.C.

Chen, G., R. Hackett, D. Walker, A. Taylor, Z. Lin, and D. Grierson. 2004. Identification of a specific isoform of tomato lipoxygenase (TomloxC) involved in the generation of fatty-acid-derived flavor compounds. Plant Physiol. 136(1):2641-2651.

Conconi, A., M. Miquel, J.A. Browse, and C.A. Ryan. 1996. Intracellular levels of free linolenic and linoleic acids increase in tomato leaves in response to wounding. Plant Physiol. 111(3):797-803.

Fauconnier, M.L., A.G. Perez, C. Sanz, and M. Marlier. 1997. Purification and characterization of tomato (Lycopersicon esculentum Mill.) hydroperoxide lyase. J. Agr. Food Chem. 45:4232-4236.

Feussner, I. and C. Wasternack. 2002. The lipoxygenase pathway. Ann. Rev. Plant Biol.53:275-297.

Frenkel,C. and J.J. Jen. 1989. Tomatoes. p. 53-73.In: N.A.M.Eskin (ed.). Quality and preservation of vegetables. CRC Press, Boca Raton, Fla.

Galliard, T. and J. Matthew. 1977. Lipoxygenase-mediated cleavage of fatty acid to carbonyl fragments in tomato fruits. Phytochemistry 16:339-343.

Gray, D.A., S. Prestage, R.S.T. Linforth, and A.J. Taylor. 1999. Fresh tomato specific fluctuations in the composition of lipoxygenase-generated C6 aldehydes. Food Chem. 64:149-155.

Grierson, D. and A.A. Kader. 1986. Fruit ripening and quality, p. 241-280. In: J.G. Atherton and J. Rudich (eds.). The tomato crop, a scientific basis for improvement. Chapman \& Hall, London,.

Griffiths, A., C. Barry, A.G. Alpuche-Solis, and D. Grierson. 1999a. Ethylene and developmental signals regulate expression of lipoxygenase genes during tomato fruit ripening. J. Expt. Bot. 50(335):793-798.

Griffiths, A., S. Prestage, R. Linforth, J.Zhang, A. Taylor, and D. Grierson. 1999b. Fruit-specific lipoxygenase suppression in antisense-transgenic tomatoes. Postharvest Biol. Technol. 17:163-173.

Heitz, T., D.R. Bergey, and C.A Ryan. 1997. A gene encoding a chloroplast-targeted lipoxygenase in tomato leaves is transiently induced by wounding, systemin, and methyl jasmonate. Plant Physiol. 114:1085-1093.

Howe, G. and C. Ryan. 1999. Suppressors of systemin signaling identify genes in the tomato wound response pathway. Genetics 153:1411-1421.
Howe, G.A., G.I. Lee, A. Itoh, L. Li, and A. DeRocher. 2000. Cytochrome P450-dependent metabolism of oxylipins in tomato: Cloning and expression of allene oxide synthase and fatty acid hydroperoxide lyase. Plant Physiol. 123:711-724.

Jandus, J., O. Valentova, J. Kas, J. Daussant, and C. Thevenot. 1997. Phospholipase D during tomato fruit ripening. Plant Physiol. Biochem. 35(2):123-128.

Li, L., C. Li, G.I. Lee, and G.A. Howe. 2002. Distinct roles for jasmonic acid synthesis and action in the systemic wound response of tomato. Proc. Nat. Acad. Sci. (USA) 99:6416-6421.

Li, C., G. Liu, C. Xu, G.I., Lee, P. Bauer, H. Ling, M. Ganal, and G.A. Howe. 2003. The tomato suppressor of prosystemin-mediated responses 2 gene encodes a fatty acid desaturase required biosynthesis of jasmonic acid and the production of a systemic wound signal for defense gene expression. Plant Cell 15:1646-1661.

Linforth, R.S., I. Savary, B. Pattenden, and A.J. Taylor. 1994. Volatile compounds found in expired air during eating of fresh tomatoes and in the headspace above tomatoes. J. Sci. Food Agr. 65:241-247.

Meilgaard, M., G.V. Civille, and B.T. Carr. 1999. Sensory evaluation techniques, 3rd ed. CRC Press, Boca Raton, Fla.

Pinhero, R.G., K.C. Almquist, Z. Novotna, and G. Paliyath. 2003. Developmental regulation of phospholipase D in tomato fruits. Plant Physiol. Biochem. 41(3):223-240.

Riley, J.C.M., C. Willemot, and J.E. Thompson. 1996. Lipoxygenase and hydroperoxide lyase activities in ripening tomato fruit. Postharvest Biol. Technol. 7:97-107.

Riley, J.C.M. and J.E. Thompson. 1998. Ripening-induced acceleration of volatile aldehyde generation following tissue disruption in tomato fruit. Physiol. Plant. 104:571-576.

Routaboul J-M, S.F. Fischer., and J. Browse. 2000. Trienoic fatty acids are required to maintain chloroplast function at low temperatures. Plant Physiol. 124:1697-1705.

Song, J., L.H. Fan, and R.M. Beaudry. 1998. Application of solid phase microextraction and gas chromatography time-of-flight mass spectrometry for rapid analysis of flavor volatiles in tomato and strawberry fruits. J. Agr. Food Chem. 46(9):3721-3726.

Suurmeijer, C.N.S.P., M. Perez-Gilabert, D.J. van Unen, H.T.W.M. van der Hijden, G.A. Veldink, and J.F.G. Vliegenthart. 2000. Purification, stabilization and characterization of tomato fatty acid hydroperoxide lyase. Phytochemistry 53:177-185.

Tandon, K.S., E.A. Baldwin, and R.L. Shewfelt. 2000. Aroma perception of individual volatile compound in fresh tomatoes (Lycopersicon esculentum Mill.) as affected by medium of evaluation. Postharvest Biol. Technol. 20:261-268.

Taylor, A.J. and R.S. Linforth. 2000. Techniques for measuring volatile release in vivo during consumption of food, p. 8-21. In: D.D. Roberts and A.J. Taylor (eds.), Flavor release. Amer. Chem. Soc. Washington D.C.

Wang, C., C.K. Chin, C.T. Ho, C.F. Hwang, J. Polashock, and C.E. Martin. 1996. Changes of fatty acids and fatty acid derived compounds by expressing the yeast $\Delta-9$ desaturase gene in tomato. J. Agr. Food Chem. 44:3399-3402.

Wang, C., J. Xing, C.K. Chin, C.T. Ho, and C.E. Martin. 2001. Modification of fatty acids change flavor volatiles in tomato leaves. Phytochemistry 58:227-232.

Whitaker, B.D., D.L Smith, and K.C. Green. 2001. Cloning, characterization and functional expression of a phospholipase D alpha cDNA from tomato fruit. Physiol. Plant. 112(1):87-94.

Yilmaz, E., K.S. Tandon, J.W. Scott, E.A. Baldwin, and R. Shewfelt. 2001. Absence of a clear relationship between lipid pathway enzymes and volatile compounds in fresh tomatoes. J. Plant Physiol. 158:1111-1116.

Zhuang, H., T.R. Hamilton-Kemp, R.A. Andersen, and D.F. Hildebrand. 1996. The impact of alteration of polyunsaturated fatty acid levels on C-6 aldehyde formation of Arabidopsis thaliana leaves. Plant Physiol. 111:805-812. 